\title{
Correction: Planetary science: Penitent Pluto
}

Luca Maltagliati

Nature Astronomy 1, 0016 (2017); published 4 January 2017; corrected 13 January 2017.

In the version of this News and Views originally published the sentence giving the penitent formation timescale was incorrect and should have read: 'Moreover, in order to explain the current height and tri-modal orientation, they infer that these features must be a few tens of millions of years old. In addition, $\mathrm{N}_{2}$ and $\mathrm{CH}_{4}$ were mistakenly displayed with superscript numbers in the HTML version. These errors have been corrected in all versions of the News and Views. 